\title{
Synthèse des observations récentes de Stenoria analis (SchAUM) (Coleoptera, Meloidae) en France et dans les régions voisines
}

\author{
Par Nicolas J Vereecken *, Pierre Dufrêne **, Johannes LüCKmanN ***, Jelle DeValez ${ }^{4 *}$, Ivo RaemaKerRS ${ }^{5 *}$ \\ et Marco A Bologna ${ }^{6 *}$
}

\begin{abstract}
Résumé. Nous présentons une synthèse des données biogéographiques de France et des régions voisines relatives à Stenoria analis (SCHAUM), un coléoptère parasite des nids de Colletes en Europe occidentale. Les données rassemblées indiquent que ce méloïdé semble avoir conquis les régions plus septentrionales très récemment, probablement en suivant l'expansion géographique récente de son hôte principal, Colletes hederae SCHMIDT \& WESTRICH.
\end{abstract}

\begin{abstract}
We provide a synthesis of records from France and neighbouring regions concerning Stenoria analis (SCHAUM), a parasitic beetle of Colletes nests in western Europe. The data gathered indicate that this blister beetle has expanded its geographic range to the north, presumably following the recent population dynamics of its primary host, Colletes hederae SCHMIDT \& WESTRICH.
\end{abstract}

Zusammenfassung. Der Ölkäfer Stenoria analis (SCHAUM) parasitiert in Westeuropa die Nester von Colletes. Die Nachweise des Käfers aus Frankreich sowie den benachbarten Ländern werden zusammenfassend dargestellt. Die Daten deuten daraufhin, dass der Käfer sein Verbreitungsgebiet nach Norden ausgeweitet hat, möglicherweise der aktuellen Populationsdynamik des primären Wirtes Colletes hederae SCHMIDT \& WESTRICH folgend.

\section{Ecologie de Stenoria analis (SGHAUM)}

Pour rappel, nos observations de terrain menées depuis 2007 ont permis d'illustrer la plupart des étapes du cycle de vie de $S$. analis, depuis l'accouplement des imagos jusqu'à l'invasion des nids de leur hôte principal, l'abeille du lierre (Colletes hederae SCHMIDT \& Westrich 1993 (Hym. Colletidae)) (Vereecken \& Mahé 2007; Mahé 2008). Le développement larvaire souterrain des larves de $S$. analis fit l'objet d'une étude remarquablement détaillée par Mayet (1875) qui réalisa ses observations dans la région de Montpellier (France).

Une fois l'accouplement terminé, les femelles de $S$. analis entreprennent l'exploration de leur environnement proche à la recherche d'un site de ponte. Les supports de prédilection sont généralement des brindilles isolées de graminées (Figures 1A, 1B) ou l'envers des feuilles de certains végétaux rampants (ronce, lierre, etc.), mais il nous est également arrivé

\footnotetext{
* Nicolas J Vereecken, Evolution Biologique \& Ecologie, Université Libre de Bruxelles CP 160/12, Av. F.D. Roosevelt 50, B-1050 Bruxelles, Belgique. E-mail: nicolas.vereecken@ulb.ac.be

** Pierre Dufrêne, 65 Rue de la Duché, F-50100 Cherbourg, France. E-mail: energi@wanadoo.fr

*** Johannes Lückmann, Leo-Grewenig Straße 3, 64625 Bensheim, Germany E-mail:JLueckmann@t-online.de

4* Jelle Devalez, Adresse actuelle: BARC-EAST, BLDG 308, RM 124 10300 Balt. Ave., Beltsville, MD 20705, USA. E-mail: jelle.devalez@gmail.com

5* Ivo Raemakers, Van Caldenborgstraat 26 NL-6247 CG Gronsveld, Pays-Bas. E-mail: ivo.raemakers@hetnet.nl

6* Marco Bologna, Dipartimento di Biologia ambientale, Università Roma Tre, Viale Marconi, 446, I-00146 Roma, Italie. E-mail: bologna@uniroma3.it
}

d'observer des pontes sur des fils barbelés utilisés comme clôture de parcelle (Mahé 2008; obs. pers.). Les oeufs sont pondus en groupes de plusieurs dizaines à plusieurs centaines d'individus, et les larves de premier stade, appelés triongulins, émergent une à deux semaines plus tard. Dès l'émergence, les triongulins sécrètent une substance soyeuse qui a pour effet de les maintenir en groupes compacts qui se détachent de leur support sous l'effet de leur masse et de leur agitation permanente. Il n'est donc pas rare d'observer de tels amas de triongulins grouillants suspendus par un fil de soie à leur support originel (Figure 1C, 1D). Les groupes de triongulins sont le plus souvent observés à proximité des sites de nidification exploités par $C$. hederae. Les mâles de l'abeille du lierre sont irrésistiblement attirés par les amas de triongulins de grande taille qu'ils approchent et avec lesquels ils entrent rapidement en contact comme s'ils tentaient de s'accoupler (Figure 1E). Certaines de nos macrophotographies de ces interactions montrent d'ailleurs les mâles de $C$. hederae approchant les amas de triongulins tout en dévoilant leur capsule génitale. Ce comportement témoigne d'une stimulation sexuelle d'origine chimique : les amas de triongulins émettent en effet des composés identiques à ceux qui constituent la phéromone sexuelle des femelles de l'abeille du lierre (Vereecken, résultats non publiés). Le seul autre cas de ce type connu dans le monde est celui d'un Meloe et de son hôte, une abeille du genre Habropoda, pour lesquels le mimétisme chimique a déjà été mis en évidence (Saul-Gershenz \& Millar 2006). Dès que le contact est établi avec les mâles de $C$. hederae, les triongulins se fixent à la pilosité qui orne principalement le thorax et la région située sous les mandibules de leur hôte (Mahé 2008), et ils profitent 


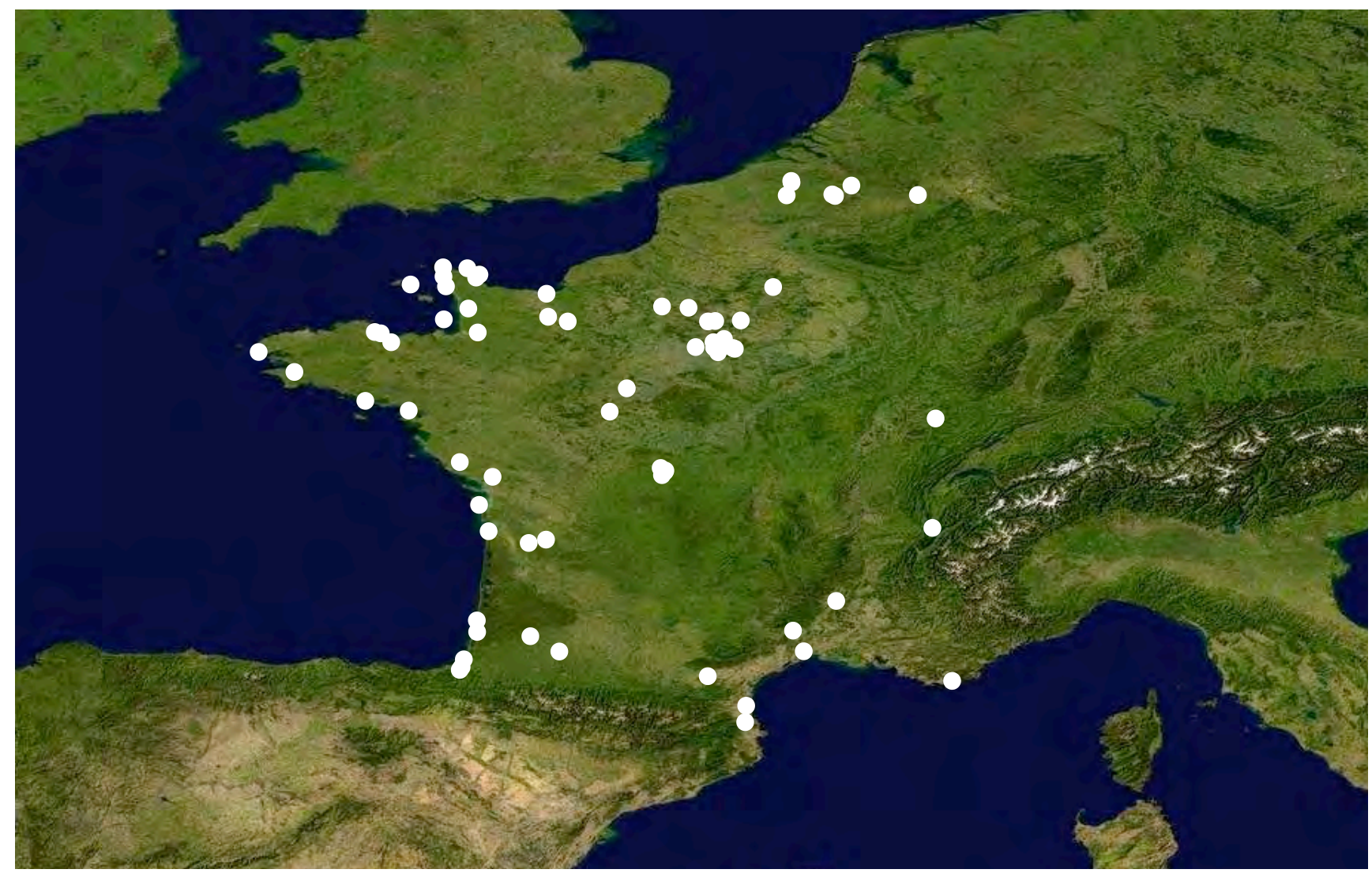

Figure 2. Synthèse des observations récentes de Stenoria analis (SCHAUM) en France et dans les régions voisines (fond de carte NASA)

emmenant sur un autre site où ils pourront interagir avec des hôtes naïs (Mahé 2008). Ce phénomène pourrait expliquer pourquoi certains auteurs comme Mayet (1875) ou Moenen (2009) n'ont pas eu l'occasion d'observer les interactions entre $C$. hederae et $S$. analis en conditions naturelles, alors que les mâles de l'abeille hôte étaient déjà activement à la recherche de leurs femelles et que les triongulins avaient probablement déjà été visités par certains mâles.

La découverte de cette interaction singulière nous a tout naturellement conduit à nous interroger sur la distribution géographique de $S$. analis et sur la dynamique possible de ses populations étant donné que son espèce hôte principale semble avoir fait preuve d'une expansion géographique considérable au cours des 10 dernières années (Vereecken et al. 2009; Burger 2010 dans ce numéro).

\section{Données biogéograpiques relatives à Stenoria analis (SGHAUM) en France et dans les régions voisines}

Bien qu'il ne soit pas rare d'observer les adultes de $S$. analis à proximité des nids de leurs hôtes, les données relatives à cette espèce sont peu nombreuses dans la région étudiée. Ce phénomène est probablement dû à un faible effort d'échantillonnage, notamment du fait de la courte vie des adultes (une à deux semaines tout au plus) et de la difficulté pour l'entomologiste de détecter des groupes de triongulins dans leur milieu naturel. Ces facteurs donnent l'illusion d'une distribution géographique fortement fragmentée.
Au cours des étés 2009 et 2010, nous avons tout d'abord entrepris de rassembler les données relatives à S. analis dans la littérature (Pasquet 1923; Duprez 1936-1945; Sorel 1989, 1992; Mineau 1994; Cantonnet et al. 1995; Livory 1998; Brustel \& Rogé 1999; Leclercq 1999; Mériguet \& Zagatti 2001; Chevin 2000; David 2003; Bouyon 2004; Mahé 2008; Moenen 2009). Parallèlement, nous avons fait appel aux membres de nombreux réseaux naturalistes afin de récolter des données plus récentes. L'ensemble de ces données biogéographiques sont présentées à la Figure 2 et indiquent que $S$. analis est une espèce nouvelle pour la Belgique et les Pays-Bas.

\section{Perspectives}

Il est intéressant de noter que la distribution géographique de l'hôte de $S$. analis, l'abeille du lierre, est principalement centrée sur la France et s'étend au nord jusqu'en Belgique et aux Pays-Bas (Vereecken et al. 2009) d'où proviennent également les données les plus septentrionales du coléoptère (Figure 2). L'absence totale de donnée historique de $S$. analis dans la moitié nord de la France suggère une installation relativement récente. Sur base des données récoltées, nous suggérons que le facteur anthropique joue probablement un rôle important dans l'expansion géographique du couple hederae-analis, notamment via la multiplication des carrières, friches anthropiques et autres grands talus routier et péri-urbains (voir aussi l'article de Burger 2010 dans ce numéro). 
En Europe, les données historiques indiquent que $S$. analis est également connu de la Péninsule Ibérique jusqu'à l'Europe Centrale et du sud de la Russie jusqu'en Turquie, en passant par l'Italie, la Croatie, le Maroc (Pardo Alcaide 1958) et la Libye (Bologna 2009). Nous n'avons pas récolté de données provenant de ces régions au cours de nos premières démarches, mais les premiers résultats illustrés ici nous ont encouragé à poursuivre les efforts d'échantillonnage, notamment via des associations entomologiques locales. A l'évidence, $S$. analis est probablement capable d'exploiter d'autres espèces de Colletes dans ces régions où $C$. hederae est absente.

Nous encourageons tous les naturalistes à se pencher sur cette espèce qui promet des perspectives d'observations au cours des prochaines saisons. Nous projetons de rassembler les données biogéographiques de $S$. analis afin de déterminer l'étendue et la dynamique de sa distribution, notamment dans les régions marginales au nord et à l'est de la distribution de C. hederae.

\section{Remerciements}

Nous remercions Rosita Moenen (Bennekom, NL), et les nombreux naturalistes qui ont eu la gentillesse de nous communiquer leur données biogéographiques relatives à Stenoria analis et nous espérons pouvoir poursuivre cette collaboration au cours des prochaines saisons avec d'autres entomologistes qui s'intéresseraient à ce coléoptère cleptoparasite.

\section{Références bibliographiques}

Bologna MA, 1991. Coleoptera Meloidae. Fauna d'Italia. XXVIII. Calderini, Bologna.

Bologna MA, 2009. The Meloidae of Lybia: an annotated catalogue and description of three new species. Annales de la Société Entomologique de France 45(3): 345-364.

Bouyon H, 2004. Catalogue des Coléoptères de l'IDF. tome 11: Tenebrioidea. Le Coléoptériste (suppl.) 7 (2): 1-53.

Brustel H \& Rogé J, 1999. Sur quelques Coléoptères rares ou peu connus du sud-ouest de la France (deuxième partie). L'Entomologiste 55(3): 91-101.

Burger R, 2010. On the presence of Colletes hederae ScHMIDT \& WEstrich and $C$. brevigena NoskiewICZ (Hymenoptera, Colletidae) in Serbia . OSMIA 4: 11-14.
Cantonnet F, Gasset L \& Toda G, 1995. Coléoptères du massif de Fontainebleau et de ses environs. Avon: Association des Naturalistes de la Vallée du Loing 251.

Ghevin H, 2000. A propos de Stenoria analis (Coleoptera Meloidae). L'Entomologiste 56(2): 82.

David CT, 2003. Land invertebrates recorded from the proposed Guernsey Ramsar site. Guernsey Biological records center 15.09.2003

Duprez R, 1936-1945. Catalogue des Coléoptères des départements de Seine inférieure et de l'Eure. Bulletin de la Société des Amis des Sciences Naturelles et du Muséum de Rouen: 1-300.

Leclercq J-P 1999. Stenoria analis (SCHAUM) dans le Finistère (Coleoptera, Meloidae). L'Entomologiste 55(6): 276.

Livory A, 1998. Faune chausiaise: une surprise de taille. Argiope 22: 13-18.

Mahé G, 2008. Observation en Loire-Atlantique (France) de Stenoria analis (SCHAUM) (Coleoptera Meloidae), cleptoparasite de Colletes hederae SCHMIDT \& WESTRICH (Hymenoptera Colletidae). OSMIA 2: $11-15$.

Mant JG, Brändli G, Vereecken NJ, Schulz G, Francke W \& Schiestl FP, 2005. Cuticular hydrocarbons as source of the sex pheromone in Colletes cunicularius (Hymenoptera: Colletidae) and the key to its mimicry by the sexually deceptive orchid Ophrys exaltata (Orchidaceae). Fournal of Chemical Ecology 31(8): 1765-1787.

Mayet V, 1875. Mémoire sur les moeurs et métamorphoses d'une nouvelle espèce de Coléoptère de la famille des Vésicants, le Sitaris colletis. Annales de la Société Entomologique de France 5: 64-92.

Mériguet B \& Zagatti P, 2001. Inventaire entomologique de la forêt régionale de Bréviande (Seine-et-Marne). Guyancourt: OPIE. 35

Mineau A, 1994. Stenoria analis (ScHAUM) en forêt de Fontainebleau (Coleoptera Meloidae). L'Entomologiste 50(1): 80.

Moenen R, 2009. Kleptoparasieten (Coleoptera: Meloidae, Hymenoptera: Apidae) bij zijdebijen (Hymenoptera: Apidae). Enomologische Berichten 69(1): 2-8.

Pardo Alcaide A, 1958. Analectas entomologicas. VII. Los Sitarini de la Peninsula Iberica (Col. Meloidae). Graellsia 16: 13-22.

Pasquet O, 1923. Coléoptères de la Manche. Mémoires de la Société des Sciences Naturelles et Mathématiques de Cherbourg: 1-350.

Sorel C, 1989. Stenoria analis (SCHAUM) dans l'Indre Coleoptera,

Meloidae). L'Entomologiste 45(4-5): 202.

Sorel C, 1992. Observations sur Stenoria analis (SCHAUM) (Coleoptera

Meloidae). L'Entomologiste 48(3): 129-132.

Saul-Gershenz LS \& Millar JG, 2006. Phoretic nest parasites use sexual deception to obtain transport to their host's nest. Proceedings of the National Academy of Sciences of the USA 103(38): 14039-14044.

Vereecken NJ \& Mahé G, 2007. Larval aggregations of the blister beetle Stenoria analis (SCHAUM) (Coleoptera: Meloidae) sexually deceive patrolling males of their host, the solitary bee Colletes hederae SCHMIDT \& WESTRICH (Hymenoptera: Colletidae). Annales de la Société Entomologique de France 43(4): 493-496.

Vereecken NJ, Schwenninger H, Gogala A \& Roberts SPM, 2009. Mise à jour de la distribution géographique de l'abeille du lierre, Colletes hederae SCHMIDT \& WESTRICH (Hymenoptera, Colletidae) en Europe. OSMIA 3: 2-3.

Villemant G, 2001. Les Coléoptères Méloïdés cleptoparasites de nids d'abeilles solitaires. Insectes 121: 7-10. 